\title{
Idiopathic ileoileal intussusception without lead point in a 4- year-old child
}

\author{
Ayşe Selcan Koç ${ }^{1}$, Zerrin Özçelik ${ }^{2}$, Feride Fatma Görgülü ${ }^{1}$ \\ ${ }^{1}$ Department of Radiology, University of Health Sciences, Adana City Hospital, Adana, Turkey \\ ${ }^{2}$ Department of Pediatric Surgery, University of Health Sciences, Adana City Hospital, Adana, Turkey
}

DOI: $10.18621 /$ eurj.375051

\begin{abstract}
Intussusception is common abdominal emergency in early childhood. Idiopathic ileoileal intussusceptions are rare in children without an apparent predisposing cause. Patients present with vomiting and colicky abdominal pain, which can mimic many conditions. We report a case with primary ileoileal intussusception, which was successfully reduced manually during exploratory laparotomy. There was no evidence enlarged mesenteric lymph nodes.
\end{abstract}

Keywords: Intussusception, childhood; ileoileal, idiopathics, lead point, laparoscopic surgery

Received: January 4, 2018; Accepted: February 27, 2018; Published Online: April 4, 2018

I ntussusception is the second most common cause of acute abdomen in early children, following appendicitis and it is located in the ileocolic, ileocecal, or ileoileocolic part of the bowel $[1,2]$. Isolated ileoileal intussusception is rare and it is frequently associated with pathological lead points while its occurrence at young children [3-5]. Presentation of small bowel intussusception is typically subacute and therefore difficult to diagnose preoperatively [5].

In this paper we present acase of a 4-year-old boy with surgically proven ileoileal intussusception without lead point.

\section{CASE PRESENTATION}

A previously healthy 4-year-old boy presented with a 2-day history of right upper-middle quadrant abdominal pain and low-grade fever. He had no nausea, vomiting or chills. According to his mother last normal bowel movement of the child was 6 hours ago, and his mother reported no urinary complaints, a history of taking any medications in the past.

On examination his abdomen was non-distended, tender to palpation and rebound on right upper-middle quadrant. He had normal active bowel sounds. There was no palpable mass and costo-vertebral angle tenderness. Rectal examination was normal with hemoccult negative brown stool. The genitourinary examination was also unremarkable.

His white blood cell (WBC) count was $3.8 \times 10^{3} / \mathrm{L}$, C-reactive protein of $0.34 \mathrm{~g} / \mathrm{L}$, erythrocyte sedimentation rate (ESR) was $8 \mathrm{~mm} / \mathrm{h}$, uric acid was $2.32 \mathrm{mg} / \mathrm{dL}$, alanine aminotransferase (ALT) was 123.3 U/L and other serum electrolytes were within normal limits. The results of other blood tests and urine analysis were within normal ranges.

Plain abdominal radiography was performed and did not show signs of obstruction or perforation (Figure 1). The abdominal axial and longitudinal

Address for correspondence: Ayşe Selcan Koç, MD., Assistant Professor, University of Health Sciences, Adana City Hospital, Department of Radiology, Adana, Turkey

E-mail:drayseselcankoc@gmail.com,Tel: +90 5062425989 


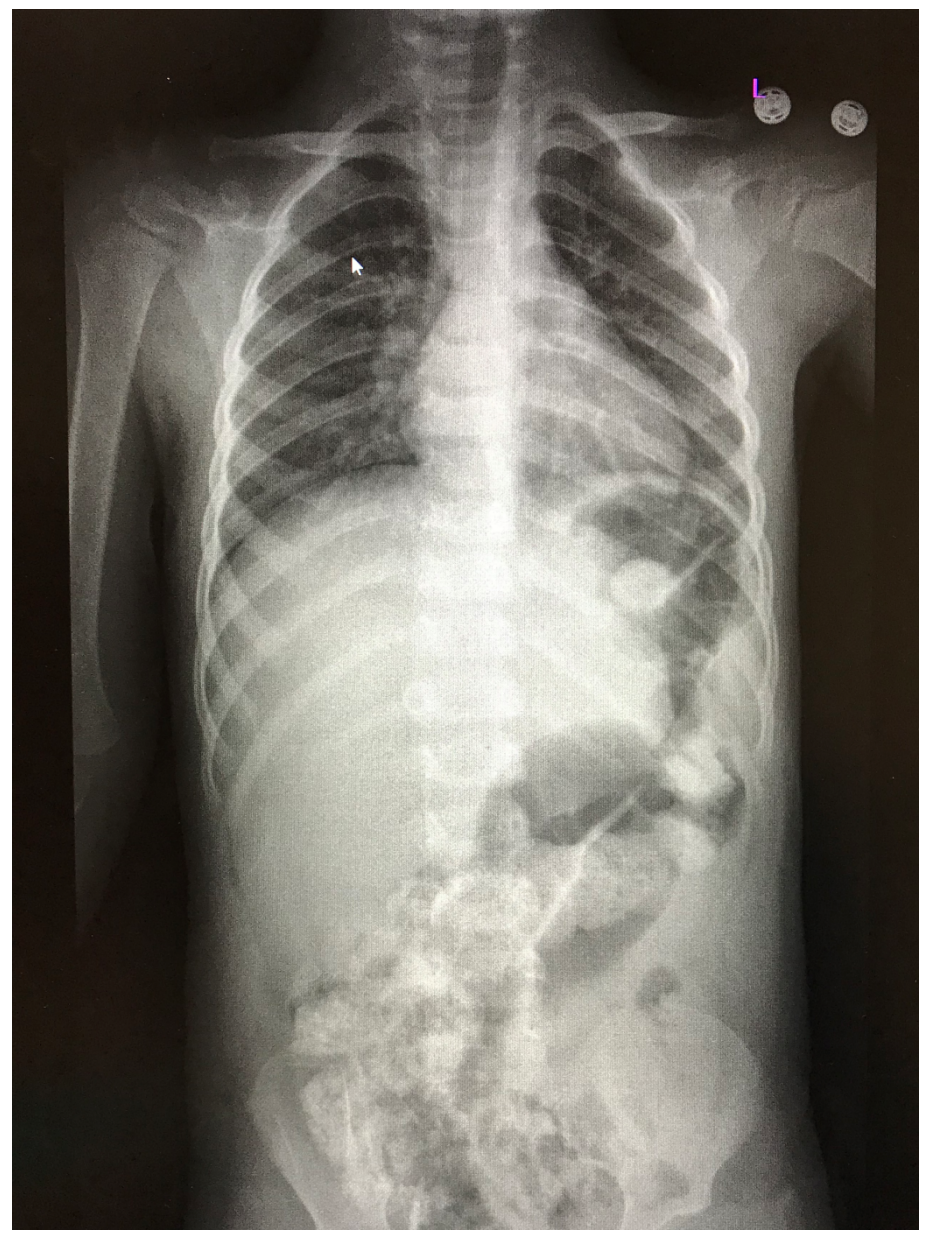

Figure 1. There are no signs of obstruction or perforation on plain abdominal radiography.

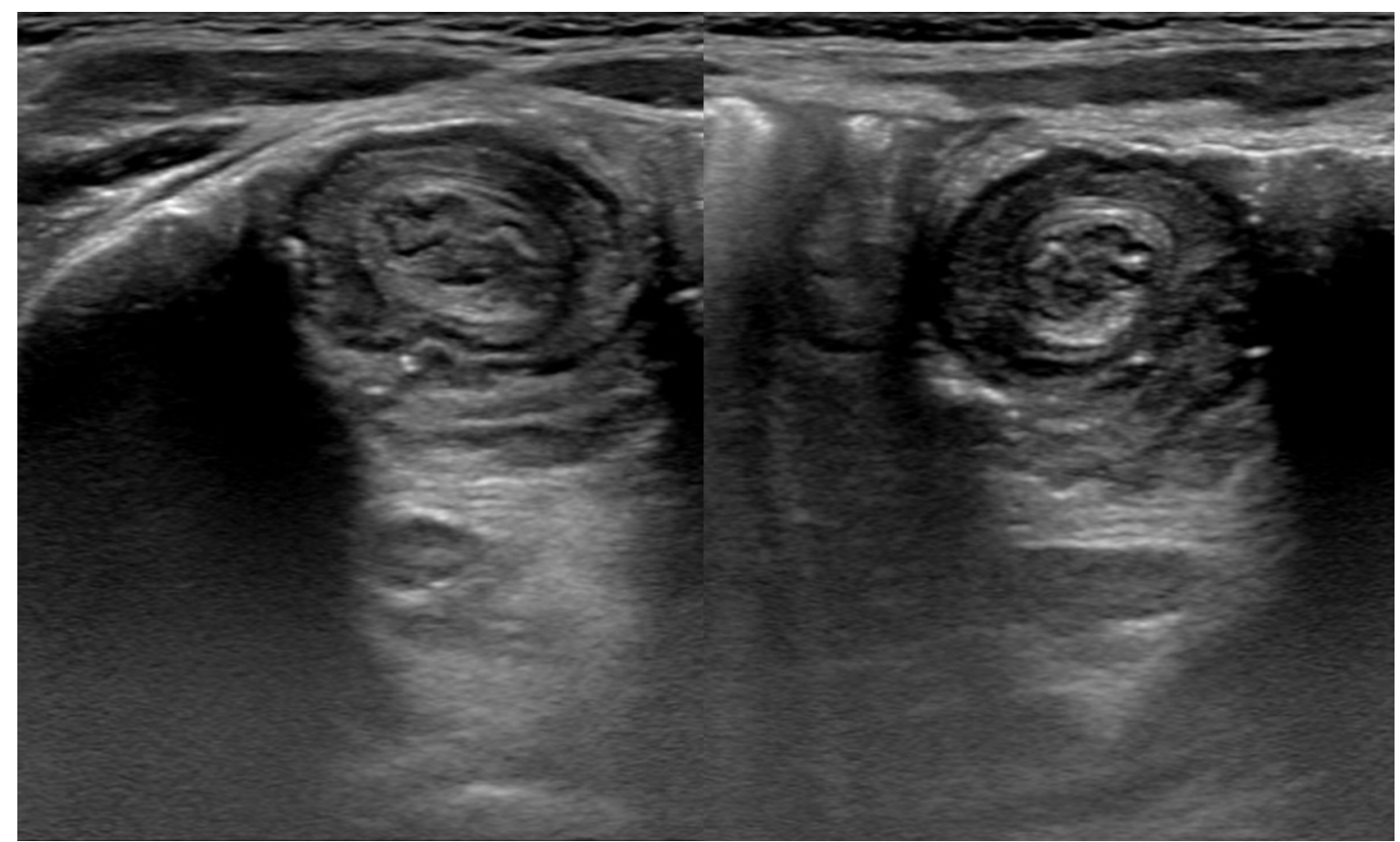

Figure 2. Abdominal ultrasonographic axial images show an increase of intestinal wall thickness like doughnut sign. 


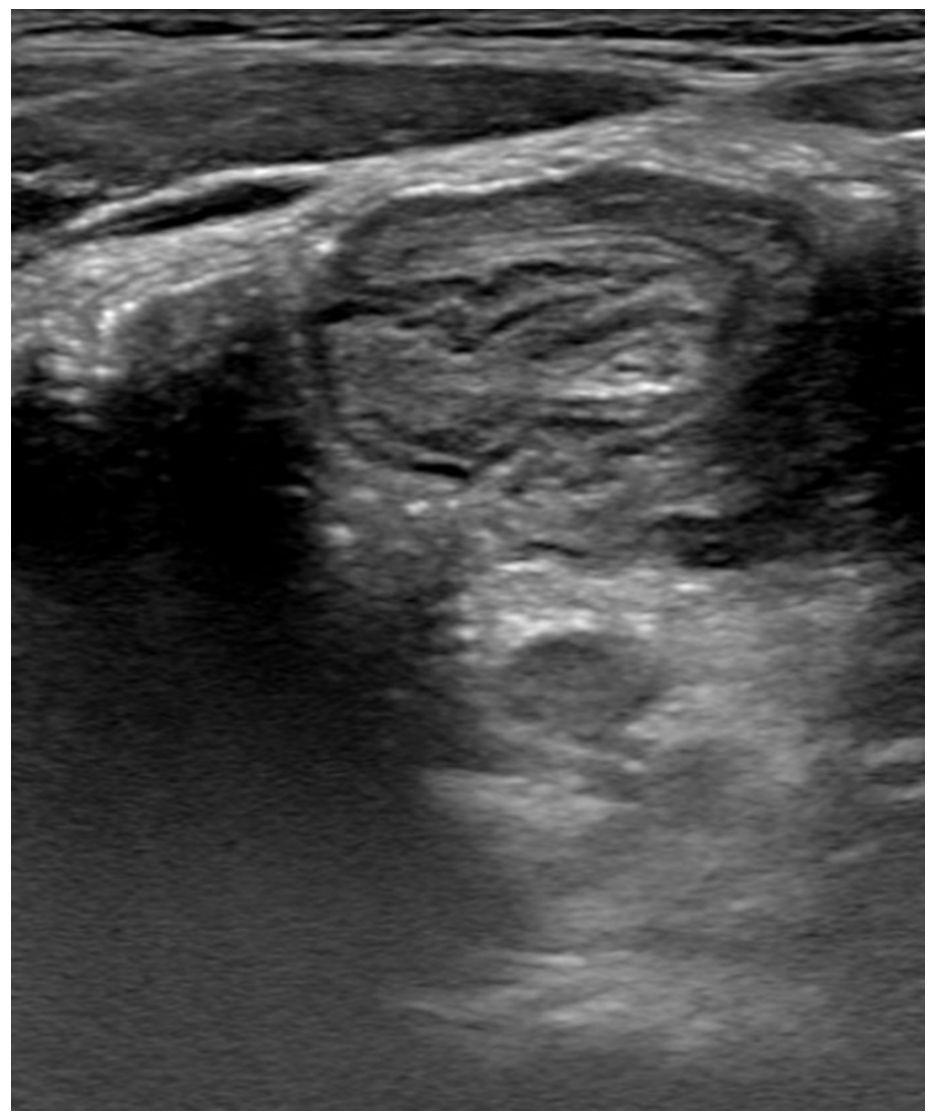

Figure 3. Abdominal ultrasound longitudinal image shows an increase of intestinal wall thickness.

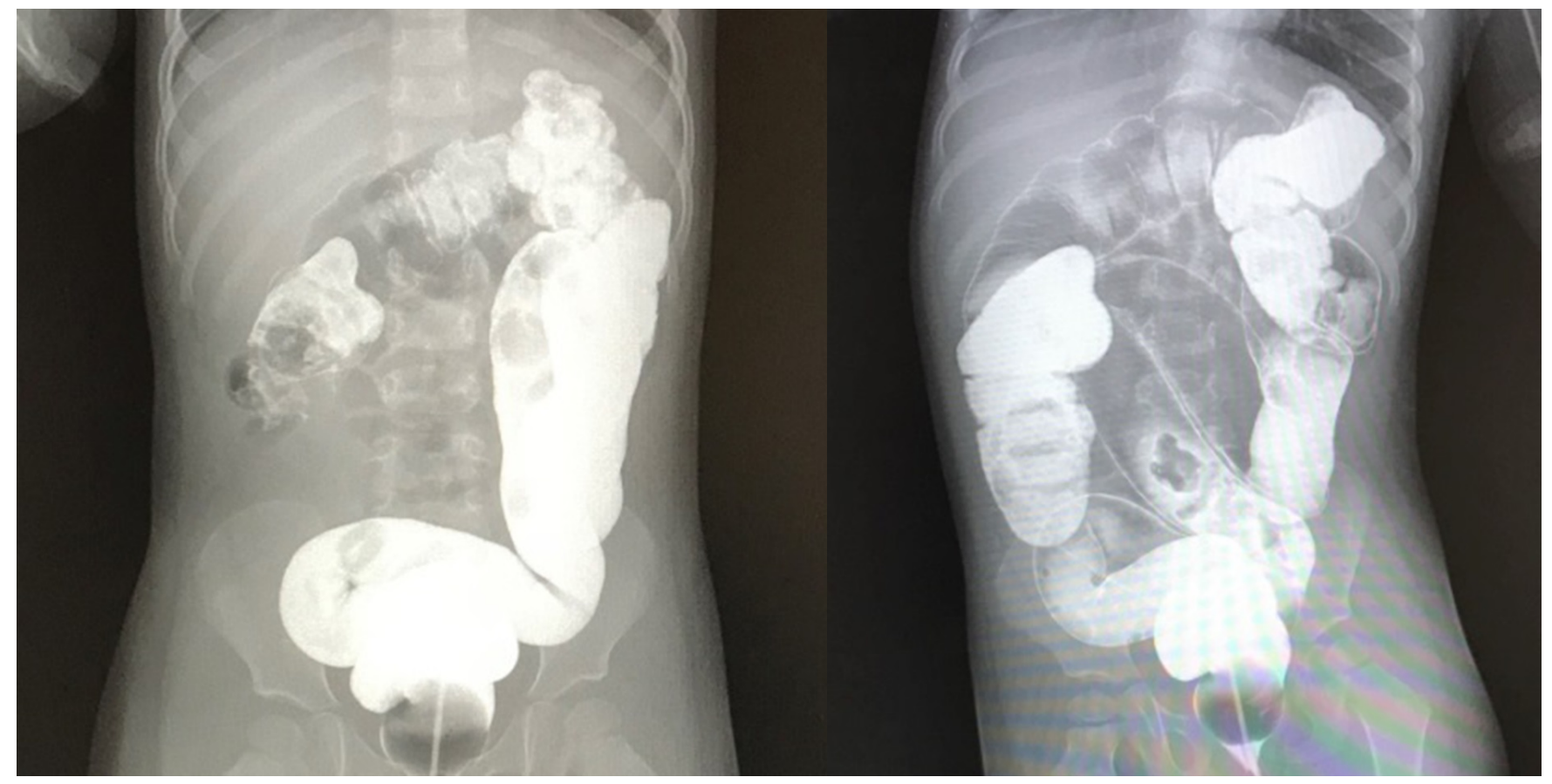

Figure 4. Hydrostatic reduction with barium enema under fluoroscopy. 
abdominal ultrasonographic images showed an increase of intestinal wall thickness like doughnut sign and the intestinal wall was measured as $2.7 \mathrm{~cm}$ at the widest point in the right upper-middle quadrant (Figures 2 and 3). Color Doppler ultrasound demonstrated no significant loss of intestinal perfusion. Hydrostatic reduction with barium enema under fluoroscopy was performed but reduction did not successfully (Figure 4).

$\mathrm{He}$ underwent exploratory laparotomy and primary ileoileal intussusception was found and manual reduction was successful. There was no evidence enlarged mesenteric lymph nodes. The postoperative recovery was uneventful.

\section{DISCUSSION}

Intussusception is the second most common cause of acute abdomen in early children, following appendicitis [1]. It occurs when a segment of intestine invaginates into the near intestinal lumen. Frequently, the terminal ileum telescopes into the colon but colocolic and ileo-ileal variants can also occur. Seventy percent of cases occur in the first year of life, with its incidence declining rapidly thereafter to $<2 \%$ in 10 15 years of age. Intussusception confined to small bowel, however, is unusual. It accounts for $1-10 \%$ of all cases of childhood intussusception, but up to $50 \%$ of cases in older children [1]. Ileoileal intussusception is more common in neonates and older children $(>5$ years old) [2]. The age and presentation of the patient plays an important role in differentiating type and pathology of the intussusception $[8,9]$.

Classic presentation of intussusceptions involves colicky abdominal pain, vomiting, palpable mass, and blood per rectum or currant jelly stools (occurring in $20 \%$ of patients) [3]. The classic clinical features of palpable abdominal mass and bloody stools are rare in small bowel intussusceptions [3, 4]. Small bowel intussusceptions usually present with vomiting and colicky abdominal pain, which can mimic many conditions [5]. Moreover, presentation of small bowel intussusception is typically subacute and therefore difficult to diagnose preoperatively. Telescoping of a segment of the intestine into the adjacent bowel loop can cause mechanical obstruction and ischemia. Early diagnosis and prompt treatment are of most importance in childhood intussusception in order to lessen discomfort and to avoid bowel ischemia or gangrene. A diagnostic ultrasound should be conducted in all school-age children presenting with acute abdominal pain [5,6]. Ultrasound is highly accurate for the diagnosis of ileo-colic intussusception with a reported sensitivity of $98 \%$ to $100 \%$; however, the diagnosis of small bowel intussusception is more difficult with an ultrasound detection rate approaching only as high as 84\% [7]. Ultrasound may illustrate small bowel intussusception as a crescent- indoughnut or multilayered 'onion skin' round mass on a transverse scan and as the short segment sandwich sign on longitudinal scan.

If treatment is delayed, cascade beginning with vascular congestion and edema of intussuscepted intestinal wall may demonstrate a highly morbid, even fatal course, with tissue ischemia, necrosis, and intestinal perforation.

In the pediatric population, treatment depends on the type of intussusception. Ileocolic intussusception, the most common type in children, requires reduction by ultrasound-guided or fluoroscopic pneumatic or hydrostatic enema, and is successful in 85 to $90 \%$ of cases $[8,9]$. Small bowel intussusception can usually be safely monitored and will reduce spontaneously without surgery [8]. Persistent small bowel intussusception, however, has been associated with a lead point or bowel necrosis, and would likely require surgical intervention [8]. Regardless of intussusception type, surgery (operative, manual reduction and/or resection or enterostomy intervention as needed) is indicated when enema reduction or close observation is unsuccessful.

Intussusception is affiliated with submucosal haemorrahage at Henoch-Schonlein purpura, cystic fibrosis, hematologic dyscrasias, postoperative changes, or lead points [10]. Other possible causes are viral inducers including rotavirus, rotavirus vaccine, and common upper respiratory illnesses. Lead points are found in $2 \%$ to $12 \%$ of children, as age increases, so does the occurrence of a lead point, and the chance of a non-surgical reduction becomes less common [10]. Unlike ileocolic intussusceptions, small bowel intussusception is frequently associated with pathological lead points (in $>29 \%$ of patients) or occurs postoperatively [7-11]. Furthermore, after 2 years of age, pathological lead points are found in one 
third of patients whereas toddler and younger children are more likely to have idiopathic intussusception [5]. In previous studies, two patients older than 2 years old with confirmed ileoileal intussusceptions had no identifiable pathologic lead point $[10,11]$. The only other case report of idiopathic intussusceptions in a teenage boy was that of a caecocolic intussusception [11]. These, together with the present case, highlight that small bowel intussusception is an important cause of abdominal pain and should be ruled out using appropriate investigations. Speculated factors predisposing to idiopathic small bowel intussusception are: swelling of small bowel wall, abnormal gastrointestinal motility, and scars or adhesions of the bowel from previous insult e.g. prior surgery $[11,12]$. The common association of ileocolic intussusceptions and lymphoid hyperplasia may lend support to the above speculation [13].

The diagnosis of intussusception in children is challenging because of a varied clinical presentation and a wide differential diagnosis. Thus it is very important to always diagnosis of intussusception and presence of any associated diseases. In our case, we used radiology and laboratory testing for diagnosis according to the general protocol. We had diagnosed intussusception without lead point through ultrasound, performed air reduction, exploratory laparotomy.

\section{CONCLUSION}

Intussusception is a relatively common and presentation of small bowel intussusception is typically sub-acute and therefore difficult to diagnose preoperatively and is easily overlooked clinically. Small bowel intussusception is frequently associated with pathological lead points. Plain X-ray and ultrasonography are important to identify cases of intussusception in children presenting with acute abdominal pain prior to surgery.

\section{Informed consent}

Written informed consent was obtained from the patient's family for the publication of this case report.

\section{Conflict of interest}

The authors declared that there are no potential conflicts of interest with respect to the research, authorship, and/or publication of this article.

\section{Acknowledgements}

We wish to thank the staff of the University of Health Sciences - Adana Health Practices and Research Center for their assistance in the management of the patient.

\section{REFERENCES}

[1] Waseem M, Rosenberg HK. Intussusception. Pediatr Emerg Care 2008;24:793-800.

[2] Ikeda T, KoshinagaT, Inoue M, Goto H, Sugitou K, Hagiwara N. Intussusception in children of school age. Pediatr Int 2007;49:58-63.

[3] Al-Salem AH. Ileoileal intussusceptions: a report of four cases. Ann Saudi Med 2000;20:310-2.

[4] Kaiser AD, Applegate KE, Ladd AP. Current success in the treatment of intussusceptions in children. Surgery 2007;142:469-75.

[5] Stringer MD, Pablot SM, Brereton RJ. Paediatric intussusceptions. Br J Surg 1992;79:867-76.

[6] Smyth R, McCallion WA, Patterson WA. Total jejunoileal intussusceptions: a case report and literature review. Ulster Med J 2009;78:10-2

[7] Applegate KE. Intussusception in children: evidence-based diagnosis and treatment. Pediatr Radiol 2009;39 (Suppl 2):S140-3.

[8] Marsicovetere P, Ivatury SJ, White B, Holubar SD. Intestinal intussusception: etiology, diagnosis, and treatment. Clin Colon Rectal Surg 2017;30:30-9.

[9] Bergman K, Mones R, Matuozzi W. Idiopathic cecolic intussusceptions in a 16-year old boy. Pediatr Surg Int 2009;25:819-21. [10] Doi O, Aoyama K, Hutson JM. Twenty-one cases of small bowel intussusceptions: the pathophysiology of idiopathic intussusceptions and the concept of benign small bowel intussusceptions. Pediatr Surg Int 2004;20:140-3.

[11] Ko SF, Lee TY, Ng SH, Wan YL, Chen MC, Tiao MM, et al. Small bowel intussusception in symptomatic pediatric patients: experiences with 19 surgically proven cases. World J Surg 2002;26:438-43.

[12] Moore SW, Kirsten M, Muller EW, Numanoglu A, Chitnis M, Le Grange E, et al. Retrospective surveillance of intussusception in South Africa, 1998-2003. J Infect Dis 2010;202 Suppl:S156-161.

[13] Yoon CS, Hur J, Kim MJ. Transient small-bowel intussusception: is it related with viral infection in young children? Biomed Imaging Interv J 2007;3: e12-e240. 